\title{
The Application of Conceptual Metaphor to English Vocabulary Teaching
}

\author{
Maoting Yin \\ Southwestern University of Finance and Economics, Chengdu 611130, Sichuan Province, China
}

\begin{abstract}
This paper considers cognitive linguistics as the guide to explore the feasibility of vocabulary teaching based on conceptual metaphor. Following the classification of conceptual metaphor, the paper probes into concrete effects of vocabulary teaching under the guidance of ontological metaphor, structural metaphor and orientational metaphor. Through detailed analysis, conceptual metaphor illuminates polysemy teaching, lexical collocation teaching and idiom teaching. Hence, it contributes to tackling the existed problems in the course of vocabulary teaching and cultivating students' competence of cognitive acquisition.
\end{abstract}

Key words: Vocabulary teaching; Ontological metaphor; Structural metaphor; Orientational metaphor

Publication date: April, 2021; Publication online: 30 April, 2021

*Corresponding author: Maoting Yin, 1749618967@qq.com

\section{Introduction}

Vocabulary acquisition has always been regarded as the most significant and challenging part of second language learning. Traditional vocabulary teaching falls into two categories: pays attention to the basic semantics of words, that is, extension or focuses on the contextual meaning of words which is connotation. However, it is strenuous to achieve desired results in both aspects. Many scholars in our country committing to vocabulary teaching have made plentiful explorations. Nevertheless, undeniable problems looms in vocabulary teaching, such as the diversity of word meaning, the complexity of vocabulary collocation and the deeper cultural meaning of vocabulary in idioms. In recent years, the development of cognitive linguistics has provided new enlightenment for English teaching. In 2004, the book Cognitive Linguistics, Second Language Acquisition and Foreign Language Teaching presents the useful enlightenment of cognitive linguistic and its related concepts to many important fields (Achard and Niemeier, 2004). Considering that conceptual metaphor is a crucial theoretical basis of cognitive linguistics, this paper discusses the application of conceptual metaphor in English vocabulary teaching, which has enlightenment to the teaching of polysemy, lexical collocation and English idioms.

\section{Application of conceptual metaphor to}

\section{polysemy teaching}

In both English and Chinese, polysemy accounts for vast majority. Lakoff (1980) points out that $70 \%$ of the everyday language is derived from metaphorical concepts and most polysemy are guided by metaphors. After continuous extension and expansion, a word derives abundant meaning which is achieved through metaphor and other means.

\subsection{Polysemy teaching by utilizing ontological metaphor}

According to Lakoff and Johnson, conceptual metaphor falls into three types: ontological metaphor, structural metaphor and orientational metaphor(Lakoff and Johnson, 1980), in which the first two contribute to various meaning of words both in Chinese and English. Ontological metaphors regard intangible concepts such as abstract and vague thoughts, feelings, mental activities, states, and events as concrete, tangible entities (Lakoff and Johnson, 1980).

Combining with ontology metaphors, teachers can strengthen students' understanding of familiar words, deepen their grasp of the metaphorical meaning and stimulate their vocabulary expansion ability. For example, "head" basically means "the uppermost part of the human body". However, we often map some abstract target domains, such as our own wisdom and capabilities to specific source domains, so "head" bears meaning "wisdom, thought, ability and emotion" in "put 
heads together" "two heads are better than one" "have a swollen head" "put something out of one's head". Another example is "heart" with its elementary meaning "the central organ of the human body". Due to its unique position in a variety of special physical and psychological activities such as "joy and sorrow", abstract target domain such as "critical thoughts and feelings" are often embodied as "heart". Students should be guided to associate and master its deeper meaning in related expressions: heartburning, heartfelt, heartsick, heartless, with all one's heart, have one's heart in one's mouth, etc. Understanding metaphorical thinking encourages students to make bold associations, actively cultivates their metaphoric ability to explore the regularity of metaphor based on basic.

\subsection{Polysemy teaching by utilizing structural metaphor}

The structural metaphor refers to cases where one concept is metaphorically structured in terms of another (Lakoff and Johnson, 1980). As exemplified in "My brain doesn't work today and I will try my best to grind out the solution", structural metaphors are used directly to describe "brain" even if "work" and "grind out" are words used to describe "machine" originally which constructs abstract things with conceptual features of "machine".

Langacker(1991) argues that the connection between the various meanings of polysemy is not arbitrary, but develops from a typical mechanism through a specific semantic extension mechanism. However, most English teachers often explain vocabulary with introducing the different meanings mechanically and ignoring the metaphorical connection. Based on structural metaphor, "foot", a frequently-used polysemy, maps "the lower part of the human body" into other structures such as "foot of the table" and "foot of the mountain". Also the structure of "theater" expands its original meaning to other real places such as "ladder classroom" and "operating room". The most classical is "bank" conveying "river bank" and "places for money transaction" which seem irrelevant. However, when the potential conceptual metaphor "MONKY IS WATER" is introduced, the function of riverbank water storage is mapped to its money storage function and money is considered a liquid that flows like water. The flow of water is the flow of funds called "money flow"; water can freeze so fund accounts is "frozen capital"; the "deposit" of water and bank funds correspond. Following the principle of cross-domain mapping, similarities have resulted in vivid expressions such as "running account" and "spend money like water". As a further extension of the savings function, "bank" can also represent "warehouse" such as "data bank" and "blood bank". The introduction of structural metaphor links the central meaning with the metaphorical meaning, thus facilitating the teaching efficiency.

\section{Application of conceptual metaphor to lexical collocation teaching}

Lexical collocation learning is a tough problem for students, which are mostly the combination of content words and particle. Orientational metaphor is a series of conceptual metaphors formed by reference to spatial orientation(Lakoff and Johnson, 1980). People project the orientation of the target domain, such as up and down, front and back, depth, center-edge, onto the abstract concept of the source domain such as emotion, physical condition, quantity, social status, etc.

\subsection{Particle teaching by utilizing orientational metaphor}

The number of particle containing ample meaning is large and when they are paired with content words, it turns more complicated. Lexical collocation is also a ubiquitous examination pattern. However, the derived meanings of particle are not irregular.

Human beings conduct production activities in nature, generating spatial orientation: up-down, south-north, out-in, come-back, etc., which reflect other abstract concepts into these basic orientational words to form metaphorical phrases. Understanding the initial meaning of particles in phrases is the semantic basis of the image patterns that represent spatial relations, it is easy to lead students to understand the potential meaning of particles. For example, "up" and "down", a pair of particles that represent opposite orientations, can form a variety of adverse metaphors. These adverbial metaphors can be used as root metaphors so that many related metaphors are derived from them. In teaching particles in sentences, teachers can first introduce orientational metaphors to students so they can accumulate $\mathrm{t}$ experience and establish mapping relationships to connect the two conceptual systems of mapping.

\subsection{Lexical collocation teaching by utilizing orientational metaphor}

Since phrases express an integral concept rather than simply adding the semantic parts, they can incur many difficulties in understanding. Orientational metaphor stems from the 
interaction between human beings and nature, hence people project concrete concepts on abstract concepts such as emotion, physical condition, quantity and social status, forming lavish linguistic expression which is conducive to lexical collocation acquisition.

By analyzing more than 600 "verb + out" and 1100 "verb + up" structures, Lindner has found that a small number of semantic structure prototypes systematically covered almost all verb- particle construction(Li Fuyin, 2004). Of the 30 most frequently used particles, "up" is the top, "down" is in the top 10, and others including "in", "out", and so on( Taylor, 2003). In the case of "up" and "down", on the basis of orientational metaphors, they can be mapped from spatial domains to more abstract domains such as physical condition, emotional state, quantity, temperature, and motion such as quantity domain (MORE IS UP, LESS IS DOWN), evaluation domain (GOOD IS UP, BAD IS DOWN), perceived domain (WARM IS UP, COLD IS DOWN) and control domain (POWER IS UP, POWERLESS-NESS IS DOWN), etc. Some content words bases on orientational metaphor such as "rise", "fall", "come", "go", etc. According to statistics, there are more than 600 such verbs in English(Wang Rongpei and Lu Xiaojuan, 1997). Phrases such as "cheer up", "let sb. down"; "build up", "run down"; "climb up", “die down"; "heat up", "cool down"; "stir up", "quite down" can be traced back to orientational metaphor.

Noun phrases can also be introduced with the help of orientational metaphors. For example, according to "MORE IS UP", "high" can be used to represent quantities such as "high price", "high speed", "high blood pressure", etc. According to the "GOOD IS UP", "high" can express positive reviews such as "high standards", "high quality", "high moral values", but also to express lofty emotional states such as "high hopes", "high ideals", etc. According to "POWER IS UP", we can find "high society", "high class", "high-status", etc. Owing that the rich meaning of particle is closely related to orientational metaphor, teachers should resort to it for particle teaching and lexical collocation and guide students to speculate and master phrase extension.

\section{Application of conceptual metaphor to idioms}

\section{teaching}

In addition to single words and phrases, the connotation of vocabulary is also beyond understanding in some sentences. People tend to remember and imitate idioms as a fixed structure, while conceptual metaphor theory holds that idioms are the product of conceptual systems so the meaning can be deduced instead of arbitrary(Lakoff and Johnson, 1980).

As a common way of language cognition among nation, conceptual metaphor provides a psychological basis for the communication of language between people of different culture. Some metaphorical words having the same cultural connotation and metaphorical meaning are called EnglishChinese cultural co-nuclear words (Schmitt N, 1997). Thus, people from different cultural backgrounds can understand conceptual metaphors by introducing conceptual metaphors into idiom learning. For example, "donkey's years" "skin and bones" "flesh and blood" and "heart and soul".

However, language can also restrict thinking. Giving that cultural traditions and social environment are disparate, divergences exist in metaphorical expression between English and Chinese languages. In metaphorical idioms, Chinese often uses a dual-source domain to map the same target domain compared with a single-source domain in English. As in “to whisper into sb's ear", "to make flesh creep", "as pretty as a flower", "to tear heart", "to end up in smoke", "with a heart of a wolf", etc. Another example is that in Chinese, "dog" contains mostly derogatory while in English dogs are often used as metaphors to praise others such as "lucky dog" and "top dog". The same is word "dragon" which is mascot in Chinese expressions but destructive monster in western culture. From the point of view of cognitive linguistics, the cultural connotation of language and thinking pattern cannot be ignored.

\section{Conclusion}

Traditional vocabulary teaching is mainly influenced by the arbitrariness of language in structuralism, ignoring the cognitive rationale of words. Consequently, most students use rote memorization to learn vocabulary, which exerts little effect. Conceptual metaphor can be used to vocabulary teaching because of its characteristics of turning abstraction into concrete, complex into concise and dreary into vivid, which conforms to our cognitive rule.

First of all, conducting polysemy teaching through ontological metaphor and structural metaphor, students can establish a contact mechanism to deduce from the basic meaning to related meanings until the marginal meaning, through which they are able to perfect the deeper meaning of familiar words and connect the seemingly irrelative meanings of polysemy. In addition, by introducing structural metaphor into phrase teaching, students can understand the 
metaphorical mechanism behind words and accept and their various collocations naturally to fully grasp the different meanings of particles. Finally, idioms can be interpreted through conceptual metaphor. However, when using the method, we should pay full attention to the culture and thinking patterns among different nationalities to correctly infer the metaphorical meaning.

To sum up, this paper points out that conceptual metaphors are closely related to vocabulary teaching. Teachers should fully realize that vocabulary covers the thinking pattern and soaks the culture of a nation. On this basis, flexible using of conceptual metaphor and other theories can guide students to replace abstract concepts with concrete and vivid metaphorical language, which can stimulate their initiative and enthusiasm.

\section{References}

[1] Achard, M \& Niemeier, s (Eds.). Cognitive Linguistics, Second Language Acquisition, and Foreign Language Teaching. Berlin: Walter de Gruyter, 2004.

[2] Lakoff, George. \& Johnson, Mark. Metaphors We Live By. Chicago: University of Chicago Press, 1980.

[3] Langacker. Foundations of Cognitive Linguistic. Stanford:Stanford University Press, 1991.

[4] Li Fuyin. Applied Cognitive Linguistics . Beijing: Chinese Literature and History Press, 2004

[5] Schmitt N, McCarthy M. Vocabulary Description, Acquisition and Pedagogy. Cambridge: Cambridge University Press, 1997.

[6] Taylor, J.R. Linguistics Categorization. Oxford: Oxford University Press, 2003.

[7] Wang Rongpei \& Lu Xiaojuan. A Survey of English Lexicology. Shanghai: Shanghai Foreign Language Education Press, 1997. 